\title{
A general framework of automorphic inflation
}

\section{Rolf Schimmrigk}

Department of Physics, Indiana University at South Bend, 1700 Mishawaka Ave. South Bend, IN 46634, U.S.A.

E-mail: rschimmr@iusb.edu

ABSTRACT: Automorphic inflation is an application of the framework of automorphic scalar field theory, based on the theory of automorphic forms and representations. In this paper the general framework of automorphic and modular inflation is described in some detail, with emphasis on the resulting stratification of the space of scalar field theories in terms of the group theoretic data associated to the shift symmetry, as well as the automorphic data that specifies the potential. The class of theories based on Eisenstein series provides a natural generalization of the model of $j$-inflation considered previously.

KEYwords: Cosmology of Theories beyond the SM, Differential and Algebraic Geometry, Discrete Symmetries

ArXiv EPrint: 1512.09082 


\section{Contents}

1 Introduction $\quad 1$

2 Automorphic inflation $\quad 2$

2.1 Multi-field dynamics 3

2.2 Definition of automorphic field theories 4

2.3 Automorphic potentials 4

$\begin{array}{lll}2.4 & \text { The kinetic term of automorphic inflation } & 7\end{array}$

3 Automorphic functions $\quad 9$

$\begin{array}{llr}3.1 & \text { Eisenstein series } & 10\end{array}$

$\begin{array}{lll}3.2 & \text { Automorphic functions of higher rank } & 11\end{array}$

4 Inflation from modular field theory $\quad 13$

$\begin{array}{lll}4.1 \text { Modular potentials } & 13\end{array}$

$\begin{array}{lll}4.2 \text { Kinetic term } & 15\end{array}$

$\begin{array}{ll}4.3 \text { Geometry of the target space } & 15\end{array}$

$\begin{array}{ll}4.4 \text { Modular Eisenstein series } & 16\end{array}$

$\begin{array}{ll}\text { 4.5 Modular functions } & 16\end{array}$

$\begin{array}{llr}5 & \text { Conclusions } & 17\end{array}$

\section{Introduction}

The space of interacting scalar fields is of interest in a variety of problems that arise in quite different fields in physics. It has however remained a somewhat amorphous object without much structure. One familiar organizing tool is to consider field multiplets $\phi^{I}, I=1, \ldots, n$, that carry representations of some continuous group $G$. Discrete groups have played a less prominent role, but have recently been considered as a way to introduce structure into the set of such theories via a stratification of this space. Theories in this framework are based on the choice of a continuous group $G$ and a discrete subgroup $\Gamma \subset G(\mathbb{Z})$ of the group $G$ considered over the rational integers $\mathbb{Z}$. Associated to such a pair of groups $(G, \Gamma)$ is a space of automorphic forms $\Phi$, viewed as functions on $G$ with certain properties. These functions descend to a curved quotient space $X$ derived from $(G, \Gamma)$ that defines the target space of the fields $\phi^{I}$. This set-up leads to a highly structured field theory space $\mathcal{F}(G, \Gamma, \Phi)$ that can be used as a starting point for models characterized by potentials $V\left(\phi^{I}\right)$ that are derived from automorphic forms on $G$, and a kinetic term determined by a target space metric $G_{I J}$ that is induced in a canonical way by the geometry of $G$.

The space $\mathcal{F}(G, \Gamma, \Phi)$ of theories can be viewed as stratified in terms of the group theoretic and automorphic characteristics when $(G, \Gamma, \Phi)$ are varied. This leads to numerical 
data that identify the different strata, including the dimension and the rank of the group $G$, the type and level of the discrete group $\Gamma$, as well as the weight of the automorphic forms $\Phi$. Depending on the specifics of the construction of $\Phi$, the automorphic structure provides additional data that characterize the field space.

Recent discussions in cosmology indicate that the basic structure of automorphic field theory defines a natural framework in which to formulate inflationary models. It has been emphasized in the literature that since inflation depends on small parameters constructed from the fundamental ingredients of the theory, phenomenological models are sensitive to the details of possible UV-completions, potentially invalidating their viability as realistic theories. An early discussion of these issues in the context of chaotic inflation [1] can be found in ref. [2]. A strategy adopted often in this context is to postulate the existence of an inflaton shift symmetry $\phi \longmapsto \phi+s_{\phi}$ as a means to protect the potential from higher order corrections. The first model to incorporate this idea was natural inflation [3, 4], but many modifications and extensions have been introduced in the intervening decades, including models that aim at realizations of this symmetry in UV-complete theories [5-8].

In the context of the shift symmetry it is natural to ask whether this ad hoc operator is part of a symmetry group that acts on the inflaton field space. The existence of such a group would provide a systematic framework in which different types of invariant potentials could be classified. A first step in this direction was outlined briefly in [9], including a concrete example of modular two-field inflation. It is the purpose of the present paper to describe the general framework of automorphic inflation as an automorphic field theory in more detail, including the discussion of classes of models with an arbitrary number of inflaton fields, leaving the phenomenological exploration of specific models to future publications. In section 2 the general structure of automorphic field theories is described, including the automorphic data that enters the construction of the inflationary potential, and the canonically constructed target space metric that is inherited from the symmetry group. Section 3 contains a discussion of a general class of automorphic forms given by Eisenstein series that can be used to construct automorphically invariant potentials. The case of the symplectic groups is used as an illustration. These groups have been of interest in the recent past in black hole physics and they provide a natural generalization of the modular group. Automorphic functions defined in terms of Eisenstein series illustrate how automorphic data provide further group theoretic information that helps to characterize the scalar field theory space. Section 4 specializes the framework of higher rank automorphic theories to the case of modular field theories, which provide an extensive framework of two-field inflation. Section 5 discusses some of the conclusions of this work, as well as some pointers to possible extensions.

\section{Automorphic inflation}

The general framework of automorphic inflation is given by multi-scalar field theories coupled to gravity and a nontrivial inflaton target space, characterized by a curved field space metric $G_{I J}$ that is inherited from the symmetry group. 


\section{$2.1 \quad$ Multi-field dynamics}

The action is given by the functional

$$
\mathcal{A}\left[\phi^{I}, G_{I J}, g_{\mu \nu}\right]=\int d^{4} x \sqrt{-g}\left(\frac{M_{\mathrm{Pl}}^{2}}{2} R-P\left(E^{I J}, V, \phi^{I}\right)\right),
$$

where $M_{\mathrm{Pl}}^{2}=1 / 8 \pi G$ is the reduced Planck mass, the signature of the spacetime metric is chosen to be $(-,+,+,+)$, and the metric $G_{I J}$ on the configuration space $X$ of the scalar field multiplet $\phi^{I}$ will be discussed in more detail below. The function $P\left(E^{I J}, V, \phi^{I}\right)$ of $E^{I J}=\frac{1}{2} g^{\mu \nu} \partial_{\mu} \phi^{I} \partial_{\nu} \phi^{J}$ and the potential $V$ is not necessarily rational. Discussions of the methods developed for the phenomenological analysis of multi-field inflation can be found in the references [10-18] and the literature cited therein.

The framework of automorphic inflation is general, but for many applications actions of the form

$$
P\left(E^{I J}, G_{I J}, \phi^{I}\right)=G_{I J} E^{I J}+V\left(F_{i}\left(\phi^{I}\right)\right),
$$

are of interest. Here the potential $V\left(F_{i}\left(\phi^{I}\right)\right)$ is taken to be a function of the inflaton multiplet $\phi^{I}$, determined by functions $F_{i}$ on the bounded domain $X$ that defines the target space of the coordinates $\phi^{I}$. The $F_{i}$ are constructed from group-theoretic automorphic forms $\Phi_{i}$.

The metric $G_{I J}$ on the target space $X$ is determined in a canonical way from the group $G$ in a way that will be described further below, leading to a Riemannian metric. The dynamics of the system $\left(g_{\mu \nu}, G_{I J}, \phi^{I}\right)$ involves the geometry of the target space as well as that of spacetime via the Einstein equations and the Klein-Gordon equation. Assuming that the covariant derivative on the inflaton space is of Levi-Civita type, the Euler-Lagrange form of the latter takes the form

$$
\square_{g} \phi^{I}+\Gamma_{J K}^{I} g^{\mu \nu} \partial_{\mu} \phi^{J} \partial_{\nu} \phi^{K}-G^{I J} V_{, J}=0,
$$

where $\square_{g}=\frac{1}{\sqrt{-g}} \partial_{\mu} \sqrt{-g} g^{\mu \nu} \partial_{\nu}$ is the d'Alembert operator and the $\Gamma_{J K}^{I}$ are the target space Christoffel symbols. This Klein-Gordon equation can be written in terms of a covariant derivative $D_{\mu}$, thought of as a combination of the spacetime Koszul connection

$$
\nabla_{\mu} \partial^{\kappa} \phi^{I}=\partial_{\mu} \partial^{\kappa} \phi^{I}+\Gamma_{\mu \nu}^{\kappa} \partial^{\nu} \phi^{I}
$$

and a contribution of the curved target space

$$
D_{\mu} \partial^{\kappa} \phi^{I}=\nabla_{\mu} \partial^{k} \phi^{I}+\left(\partial_{\mu} \phi^{J}\right) \Gamma_{J K}^{I} \partial^{\kappa} \phi^{K}
$$

as

$$
D_{\mu}\left(\partial^{\mu} \phi^{I}\right)-G^{I J} V_{, J}=0 .
$$

In a spatially flat Friedman-Lemaitre background the scalar field dynamics takes the form

$$
D_{t} \dot{\phi}^{I}+3 H \dot{\phi}^{I}+G^{I J} V_{, J}=0
$$

where $D_{t}$ is the covariant derivative in target space given by $D_{t} A^{I}=\partial_{t} A^{I}+\Gamma_{J K}^{I} \dot{\phi}^{J} A^{K}$. The Hubble parameter is constrained in terms of the energy density $\rho=\frac{1}{2} G_{I J} \dot{\phi}^{I} \dot{\phi}^{J}+V$ via $H^{2}=\rho / 3 M_{\mathrm{Pl}}^{2}$ and $\dot{H}=-G_{I J} \dot{\phi}^{I} \dot{\phi}^{J} / 2 M_{\mathrm{Pl}}^{2}$. Covariant formulations of perturbations can be found in $[14,19]$, for example. 


\subsection{Definition of automorphic field theories}

The notion of automorphic field theory derives from the idea to consider dynamical systems whose structure is constrained by both continuous and discrete symmetry groups in a systematic way. The original motivation to introduce such theories arose in the context of the shift symmetry, a discrete symmetry imposed in the inflationary context in order to suppress higher dimension operator corrections of phenomenological parameters, but the fundamental structure is independent of such applications and the framework can be formulated on its own terms, leading to the concept of automorphic field theories.

The classical concept of an automorphic function was originally introduced in the late 19th century by Klein [20] in the context of the group $G=\operatorname{SL}(2, \mathbb{R})$ and its various discrete subgroups $\Gamma \subset \mathrm{SL}(2, \mathbb{Z})$. This framework is too narrow for general multi-field inflation, which motivates to consider extensions of this framework. In the early 20th century first generalizations by Hilbert, Siegel and Maaß led to the introduction of generalized modular forms on higher-dimensional domains, as well as non-holomorphic forms, and since the 1950s work in this direction has experienced a vast generalization in the hands of Bochner, Selberg, Harish-Chandra, Langlands, and many others. As a result the concept has become less precise and different authors define it differently. Given the new conceptual picture developed for the general framework, it makes sense to distinguish between the classical case associated to the group $\operatorname{SL}(2, \mathbb{R})$ (and $\operatorname{GL}(2, \mathbb{R})$ ), which leads to two-field inflation, and the case of higher dimensional groups $G$ that are needed for true multi-field inflation. In the following the former case will be designated as modular, independently of the type of discrete groups $\Gamma$ considered, and including the non-holomorphic forms of Maaß, and the case of higher dimensional groups $G$ will be called automorphic.

A natural class of groups that could be considered in this context are semi-simple Lie groups, familiar from different fields in physics, but in order to include the general linear group GL $(n, \mathbb{R})$, ubiquitous in the context of automorphic forms and representation theory, it is useful to consider the more general framework of reductive groups.

\subsection{Automorphic potentials}

The fundamental assumption in automorphic field theory is that the potentials $V\left(\phi^{I}\right)$ are invariant under some discrete symmetry group $\Gamma$ acting on the scalar fields. In practice such functions are constructed in terms of group theoretic automorphic forms $\Phi_{i}$ and associated forms $F_{i}$ on the inflaton target space. These concepts will be made explicit in the following.

An automorphic form $\Phi$ is an object that here will be taken to be a scalar function

$$
\Phi: G \longrightarrow \mathbb{C}
$$

on a reductive linear algebraic group, i.e. a group that can be embedded into the general linear group $G \longrightarrow \mathrm{GL}(n)$. These functions are constructed in such a way as to admit a descent to the quotient space $X=G / K \cdot A$ of the reductive group $G$ with respect to a maximal compact subgroup $K$ and the split component $A$, leading to a scalar form $F: X \longrightarrow \mathbb{C}$ on the bounded domain $X$. 
The inflaton multiplet $\phi^{I}$ is defined by spacetime functions that take values in $X$

$$
\phi^{I}: M \longrightarrow X,
$$

defining coordinates on the target space. A general inflationary potential $V$ can be viewed as a map on the configuration space $\mathcal{C}^{\infty}(M, X)$ of all maps of the type (2.9)

$$
V: \mathcal{C}^{\infty}(M, X) \longrightarrow C^{\infty}(M, \mathbb{R}) .
$$

In this set-up the potentials $V\left(\phi^{I}\right)$ of the multiplet inflaton $\phi^{I}$ are defined as $V\left(F_{i}\left(\phi^{I}\right)\right)$.

Automorphic forms $F$ on the bounded domain $X$ can be constructed in terms of a special class of smooth group functions $\Phi$ on the algebraic reductive group with maximal compact subgroup $K$ relative to a discrete subgroup $\Gamma \subset G(\mathbb{Z})$. Different types of such discrete groups have been considered, in particular lattice subgroups, i.e. groups for which the quotient $\Gamma \backslash G$ has finite volume, but it is most convenient to assume that $\Gamma$ is an arithmetic subgroup, i.e. a discrete group that is commensurable to $G(\mathbb{Z})$. If $\Gamma$ is a subgroup of $G(\mathbb{Z})$ then this implies that it has finite index in $G(\mathbb{Z})$. If the group $G$ is semi-simple and of rank larger than unity then there is no distinction between lattices and arithmetic groups, but in general this is not case. The descent from the group $G$ to the domain is obtained by considering coset spaces of $G$ with respect to a maximal compact subgroup $K$, leading to a group theoretic input given by triplets $(G, \Gamma, K)$.

Automorphic forms associated to group triples $(G, K, \Gamma)$ are functions which can be characterized by a number of constraints that are mostly concerned with the transformation behavior of $\Phi$. A further condition is usually formulated in terms of the universal enveloping algebra $\mathrm{U}(\mathfrak{g})$ associated to the Lie algebra $\mathfrak{g}$ by considering the quotient $T(\mathfrak{g}) / I(\mathfrak{g})$ of the tensor algebra $T(\mathfrak{g})$ of $\mathfrak{g}$ with respect to the two-sided ideal $I(\mathfrak{g})$ generated by $V \otimes W-W \otimes$ $V-[V, W]$, for $V, W \in \mathfrak{g}$. This constraint can alternatively be viewed as a property of $\Phi$ encoded in terms of the algebra $D(G)$ of differential operators because $U(\mathfrak{g})$ and $D(G)$ are isomorphic [21]. This follows via the iteration of the basic map that associates a differential operator $D_{V}$ on functions $\Phi \in C^{\infty}(G)$ to an element $V$ of the Lie algebra $\mathfrak{g}$

$$
\mathfrak{g} \ni V \longmapsto D_{V} \Phi(g):=\left.\frac{d}{d t} \Phi\left(g e^{t V}\right)\right|_{t=0} .
$$

The most important part of the universal enveloping algebra is its center $Z_{U}$, for which the map (2.11) induces an isomorphism between $Z_{U}$ and the subspace of bi-invariant differential operators in $D(G)$. Briefly, an automorphic form on $G$ is then defined as a $\Gamma$-covariant, $Z_{G}$-covariant, $K$-finite, and $Z_{U}$-finite smooth function which satisfies a growth constraint. In more detail this entails the following.

1. The first constraint encodes $\Gamma$-covariance, the requirement that $\Phi$ transforms under a fixed arithmetic group $\Gamma \subset G$ via a character $\epsilon$ of $\Gamma$, i.e.

$$
\Phi(\gamma g)=\epsilon(\gamma) \Phi(g), \quad \gamma \in \Gamma, g \in G .
$$

2. Relative to the center $Z_{G}$ of the group $G$ the function $\Phi$ transforms in terms of the central character $\omega$

$$
\Phi(a g)=\omega(a) \Phi(g), \quad a \in Z, g \in G
$$


3. The requirement of $K$-finiteness demands that the space of functions spanned by transporting $\Phi$ via the right translation of the compact group $K$ is finite dimensional

$$
\operatorname{dim}\langle\Phi(g k)\rangle_{k \in K}<\infty
$$

4. The fourth constraint is concerned with the universal enveloping algebra $U(\mathfrak{g})$ associated to the Lie algebra $\mathfrak{g}$ of $G$. The requirement here is $Z_{U}$ finiteness, which demands that the action of the center $Z_{U}$ of $\mathrm{U}(\mathfrak{g})$ generates a finite dimensional function subspace

$$
\operatorname{dim}\langle D \Phi\rangle_{D \in Z_{U}}<\infty .
$$

Essentially this constraint generalizes the idea of considering eigenfunctions of the Laplacian of the group.

5. The automorphic form satisfies a growth constraint. There exists a norm $\|\cdot\|$ on $G$ such that

$$
|\Phi(g)| \leq C|| g \|^{n}
$$

for all $g \in G$, where $C$ is a constant and $n \geq 0$ is an integer which depends on the choice of the norm $\|\cdot\|$. If this is satisfied the function $\Phi$ is said to be of moderate growth.

6. $\Phi$ is called a cusp form if

$$
\int_{(\Gamma \cap N) \backslash N} \Phi(n g) d n=0
$$

for all $g \in G$ and all parabolic subgroups $P \subset G$ with Levi decomposition $P=M N$, where $N$ is the unipotent radical of $P$ and $M$ is called the Levi factor.

More details can be found in refs. [22-25].

Given a group function $\Phi$ of the type just outlined, it is possible to construct the descent automorphic form $F$ on the quotient $X=G / K$ which transforms under the discrete group in a way that generalizes the behavior of classical modular forms. To do so one first notes that there exists a base point $z_{0}$ in $G / K$ such that the whole quotient is obtained via the action of the group $G$, i.e. $X=\left\{g z_{0} \mid g \in G\right\}$. The descent form $F$ can then be defined at any point $X \ni z=g z_{0}$ via

$$
F(z)=J\left(g, z_{0}\right) \Phi(g),
$$

where $J\left(g, z_{0}\right)$ is a 1 -cocycle function

$$
J: G \times X \longrightarrow \mathbb{C}
$$

which satisfies

$$
J\left(g_{1} g_{2}, z\right)=J\left(g_{1}, g_{2} z\right) J\left(g_{2}, z\right) .
$$

The automorphy factor was introduced to generalize the older notion of transformations determined by powers of the Jacobian [26]. 


\subsection{The kinetic term of automorphic inflation}

The class of automorphic potentials introduced above leads to target spaces $X$ spanned by the scalar field multiplets $\phi^{I}, I=1, \ldots, n$ that are homogeneous, i.e. spaces for which group of isometries acts transitively, i.e. any two points can be related by a group element. The metric $G_{I J}$ on $X$ is taken to be induced by the left-invariant metric of the group $G$. The left translation $L_{h}$ on $G$ for an arbitrary $h \in G$, defined by $L_{g} h=h g$, can be used to induce a metric on $G$ by using the differential $d L_{g}: T_{e} G \longrightarrow T_{g} G$ to transport tangent vectors at the identity $e$ of $G$ to any other point $g$ of $G$. It therefore can be used to extend any scalar product $\langle\cdot, \cdot\rangle_{e}$ on $T_{e} G$ to the group via

$$
\langle V, W\rangle_{g}=\left\langle d L_{g^{-1}} V, d L_{g^{-1}} W\right\rangle_{e},
$$

for $V, W \in T_{g} G$. Since the tangent space $T_{e} G$ at the identity is isomorphic to the Lie algebra $\mathfrak{g}$ of $G$ via the map

$$
V_{g}(f)=\frac{d}{d t} f\left(g e^{t V}\right),
$$

where $V$ is an element in the Lie algebra $\mathfrak{g}$, it is natural to define the scalar product on the tangent space $T_{e} G$ at the identity via the Cartan-Killing form $B(X, Y)$, defined in terms of the adjoint representation $\operatorname{ad}_{V}: \mathfrak{g} \longrightarrow \mathfrak{g}$ given by $W \longmapsto \operatorname{ad}_{V} W:=[V, W]$, as

$$
B(V, W)=\operatorname{tr}\left(\operatorname{ad}_{V} \operatorname{ad}_{W}\right) .
$$

The descent of the Cartan-Killing form induced metric on $G$ to the quotient $X=$ $G / K$ can be constructed by considering the decomposition of the Lie algebra $\mathfrak{g}$ into the Lie algebra $\mathfrak{k}$ associated to the maximal compact subgroup $K \subset G$ and the orthogonal complement $\mathfrak{p}$

$$
\mathfrak{g}=\mathfrak{p} \oplus \mathfrak{k},
$$

with respect to the form $B$. The subalgebra $\mathfrak{p}$ is isomorphic to the tangent space at $z_{0}=e K=K$, and the restriction of $B$ to $\mathfrak{p}$ leads to an inner product on $\mathfrak{p} \cong T_{z_{0}} X$, which can be transported with the left translation on the quotient space $\ell_{h}: G / K \longrightarrow G / K$ given by $\ell_{h} g K=h g K$. This leads to

$$
\langle U, V\rangle_{z}=\left\langle d \ell_{g^{-1}} U, d \ell_{g^{-1}} V\right\rangle_{z_{0}} .
$$

For matrix groups an explicit realization of the quotient space can be obtained by mapping the equivalence class $g K$ to $z=g\left(g^{t}\right)$ since $K$ is the kernel of this map. This leads to a more immediate form of the scalar product as

$$
\langle U, V\rangle_{z}=\operatorname{tr}\left(z^{-1} U z^{-1} V\right) .
$$

The metric associated to this scalar product is given by

$$
d s^{2}=\operatorname{tr}\left(z^{-1} d z z^{-1} d z\right) .
$$

The local form $G_{I J}$ of the metric on $X$ can now be constructed by introducing coordinates on the quotient space that are inherited by the Iwawasa decomposition of the 
group $G$. This can be obtained from the Cartan decomposition of the Lie algebra $\mathfrak{g}=\mathfrak{k} \oplus \mathfrak{p}$ by choosing a maximal abelian subspace $\mathfrak{a}$ of $\mathfrak{p}$. The decomposition of $g \in G$ then is induced by the exponential of the Lie algebra decomposition

$$
\mathfrak{g}=\mathfrak{n} \oplus \mathfrak{a} \oplus \mathfrak{k},
$$

leading to $G=N A K$, where $N, A, K$ are the groups obtained from $\mathfrak{n}, \mathfrak{a}, \mathfrak{k}$ respectively. The quotient space is therefore spanned by $X \cong N A$ and the coordinates of $X$ can be chosen to be those that appear in $N$ and $A$.

There are several classes of Lie groups that can be used to construct automorphic field theories, including the semi-simple groups, but also reductive groups. A concrete sequence of groups which illustrates the above abstract discussion are the symplectic groups $\operatorname{Sp}(n, \mathbb{R})$. These groups most naturally generalize the model of $j$-inflation considered in [9] as an example of modular inflation is given by the symplectic groups $\operatorname{Sp}(n, \mathbb{R})$, defined as the set of $(2 n \times 2 n)$-matrices $M$ such that $[27,28]$

$$
M^{t} J M=J, \quad J=\left(\begin{array}{cc}
0 & \mathbf{1}_{n} \\
-\mathbf{1}_{n} & 0
\end{array}\right),
$$

where $\mathbf{1}_{n}$ is the $(n \times n)$-unit matrix and $M^{t}$ denotes the transpose of $M$. Writing $M$ in terms of $(n \times n)$-block matrices

$$
M=\left(\begin{array}{ll}
A & B \\
C & D
\end{array}\right) \in \operatorname{Sp}(n, \mathbb{R})
$$

translates the defining relation into the more transparent constraints

$$
\begin{aligned}
A^{t} D-C^{t} B & =\mathbf{1}_{n} \\
A^{t} C & =C^{t} A \\
B^{t} D & =D^{t} B .
\end{aligned}
$$

For $n=1$ this specializes to the modular group $\operatorname{SL}(2, \mathbb{R})$. The Iwasawa decomposition of $\operatorname{Sp}(n, \mathbb{R})$ is given by the compact subgroup $K_{G}=O(2 n, \mathbb{R}) \cap \operatorname{Sp}(n, \mathbb{R})$, which is isomorphic to

$$
K_{G} \cong\left\{\left(\begin{array}{cc}
A & B \\
-B & A
\end{array}\right) \mid A^{t} A+B^{t} B=1, \quad A^{t} B=B^{t} A\right\}
$$

the block-diagonal subgroup

$$
A_{G}=\left\{\left(\begin{array}{cc}
A & 0 \\
0 & A^{-1}
\end{array}\right) \mid A \text { diagonal with positive elements }\right\},
$$

and

$$
N_{G}=\left\{\left(\begin{array}{cc}
A & B \\
0 & \left(A^{t}\right)^{-1}
\end{array}\right) \mid A \text { upper triangular with 1s in the diagonal, } A B^{t}=B A^{t}\right\} .
$$


The compactness of $K_{G}$ can be seen by mapping it into $\mathrm{U}(n)$ via

$$
\left(\begin{array}{cc}
A & B \\
-B & A
\end{array}\right) \longmapsto A+i B .
$$

The Cartan-Killing form leads to

$$
B(V, W)=2(n+1) \operatorname{tr} V W
$$

and the local form of the metric on the target space $X=\operatorname{Sp}(n) / U(n)$ then is given modulo a factor by

$$
d s^{2}=\operatorname{tr}\left(C^{-1} d C\right)^{2}+\operatorname{tr}\left(C^{-1} d D\right)^{2}
$$

which for $n=1$ specializes to the Poincaré metric of modular inflation discussed below. This metric is usually traced, without the group theoretic interpretation, to Siegel's paper [29], but can be found in an earlier paper by Maaß [30]. The map from $G$ to $X$ can be made explicit by choosing the maximal compact subgroup $K$ to be the isotropy group of a point $x_{0} \in X$, leading to $g x_{0}=n a k x_{0}=n_{a} x_{0}$.

Automorphic forms associated to the symplectic group have played an important role in the understanding of the microscopic structure of the entropy of certain types of supersymmetric black holes (refs. [31, 32] contain reviews). Such forms can be obtained by lifting classical modular forms to Siegel forms and for the models considered so far the origin can in fact be traced to elliptic modular forms [33].

The same metric (2.37) is obtained for the target spaces $X=G / K$ with $G=\operatorname{SL}(n)$ and $K=\mathrm{SO}(n)$, where $A$ is given as above and

$$
N=\left\{\left(\begin{array}{ll}
1 & D \\
0 & 1
\end{array}\right)\right\}
$$

where $D$ is symmetric.

\section{Automorphic functions}

As noted earlier, automorphic inflation is based on the idea to consider potentials $V\left(\phi^{I}\right)$ that are invariant with respect to the action of a discrete subgroup $\Gamma \subset G$. In principle one might ask for a direct classification of the space of meromorphic functions on the target space that are invariant with respect to $\Gamma$. A more practical and natural way to construct such functions is by considering quotients of automorphic forms of the same weight with respect to $\Gamma$. A special, but systematic, class of such higher weight forms that can be used to construct quotients is comprised of Eisenstein series associated to pairs $(G, \Gamma)$, specified by further data. 


\subsection{Eisenstein series}

In their classical incarnation Eisenstein series reach back into the 19th century in the context of elliptic geometry and modular forms associated to the modular group $\operatorname{SL}(2, \mathbb{Z})$. These objects are useful for modular inflation, providing in particular the ingredients for the potential used in the two-field model of $j$-inflation, an example of modular inflation introduced in [9]. The classical series were generalized to the automorphic framework by several authors in the 1950s and 60s, in particular Selberg and Langlands, with a more detailed account published by Harish-Chandra [24]. An extensive account of Eisenstein series in the adelic framework can be found in [34]. While the adelic language does have advantages for certain questions, and much of the modern literature on automorphic forms and representations is written in it [35], this formulation is not always the best approach because it can obscure the geometric structures present in certain physical problems. The present description continues to use the archimedean framework to briefly outline the construction of Eisenstein series associated to higher rank groups, necessary for inflation with more than two fields.

Roughly speaking, Eisenstein series are obtained by combining two basic constructions, the lift of functions defined on subgroups of $G$, and an averaging process of the resulting functions over quotients of discrete subgroups. The subgroups that enter the lift can be motivated as follows. If $G$ is a reductive algebraic group the Cartan decomposition allows to factorize the group as $G=P K$, where $K$ is a maximal subgroup. The remaining factor $P$ is not reductive, hence it contains a non-trivial unipotent radical $N$. Factoring further $P=M N$ thus produces another, smaller, reductive group $M$, called the Levi factor. Defining a class of functions $I_{s}(G)$ by their transformation laws, which can be made explicit more easily in various special cases, the construction of $f_{s}$ depends in particular on the nature of the parabolic group $P$, which is encoded in the dimension of the parameter $s \in \mathbb{C}^{r}$. For maximal parabolic groups $s \in \mathbb{C}$, while for minimal $P$ the dimension $r$ of $s$ is given by the degree of the matrix group. Special cases can be found in Langlands [36] and the modular case will be described in more detail later in this paper as a framework for two-field inflation.

The common feature of all cases is that certain "root" functions $f_{M}, f_{K}$ enter that are defined on the Levi component $M$ and the maximal compact subgroup $K$, respectively

$$
f_{M}: M \longrightarrow \mathbb{C}, \quad f_{K}: K \longrightarrow \mathbb{C} .
$$

These functions are extended trivially to $G$ and twisted by a character $\chi_{s}$ on the Levi component $\chi_{s}: M \longrightarrow \mathbb{R}$, which is also extended trivially. In the maximal case, i.e. for $s \in \mathbb{C}$, the character $\chi_{s}$ can be obtained as the $s^{\text {th }}$ power of the canonically defined modular character, $\delta_{P}: P \longrightarrow \mathbb{R}^{+}$, which in this context is introduced to mediate between the left and right Haar measures on $G$, i.e. $d_{R} g=\delta_{P}(g) d_{L} g$.

Given the ingredients $\left(\chi_{s}, f_{M}, f_{K}\right)$, the lift of the functions on the smaller groups, in particular the smaller reductive group given by the Levi component $M$, to the full group $G$ is obtained by defining

$$
f_{s}(g)=\chi_{s}(m) f_{M}(m) f_{K}(k)
$$


where $g=n m k$. The Eisenstein series $E_{s, f_{P}, f_{K}}$ are defined by averaging $f_{s}$ over quotients of discrete groups where $P$ and $G$ are considered over the ring of integers $\mathbb{Z}$ as

$$
E_{s, f_{M}, f_{K}}(g)=\sum_{\gamma \in P(\mathbb{Z}) \backslash G(\mathbb{Z})} f_{s}(\gamma g) .
$$

This construction of Eisenstein series associated to reductive groups $G$ includes the holomorphic Eisenstein series associated to the modular group $\operatorname{SL}(2, \mathbb{R})$, as well as the realanalytic Eisenstein series of $\mathrm{SL}(2, \mathbb{R})$ introduced by Maaß.

The shift symmetry of the inflaton can be viewed as one of the two generators of the modular group $\mathrm{SL}(2, \mathbb{Z})$ and therefore leads to the consideration of modular inflation as a framework for two-field inflationary models. The modular group can be embedded into higher rank discrete groups $\Gamma \subset G(\mathbb{Z})$, such as subgroups of the general linear groups $\mathrm{GL}(n, \mathbb{Z})$, or the symplectic groups $\operatorname{Sp}(n, \mathbb{Z})$, and thus allows an embedding of the shift symmetry in higher rank inflation. The resulting group structured spaces of scalar field theories are stratified further via the characteristics of automorphic forms, such as their rank, weight, and level.

\subsection{Automorphic functions of higher rank}

As noted above in the geometric context of the kinetic term, the sequence of symplectic groups $\operatorname{Sp}(n, \mathbb{R})$ provides a natural extension of the modular group $\mathrm{SL}(2, \mathbb{R})$, leading to automorphic inflation based on Siegel modular forms.

The symplectic group theoretic Eisenstein series were considered originally by Siegel $[37,38]$. The domain $X$ in this case has dimension

$$
\operatorname{dim} \mathcal{H}_{n}=\operatorname{dim} \operatorname{Sp}(n, \mathbb{R}) / \mathrm{U}(n)=\frac{n}{2}(n+1),
$$

and is isomorphic to the Siegel upper halfplane defined as

$$
\mathcal{H}_{n}=\{Z=X+i Y \mid Z \text { symmetric, } Y>0\},
$$

where $Y>0$ means that $Y$ leads to a positve definite quadratic form. The symplectic group, with elements $M$ written as in (2.30), acts on $Z \in \mathcal{H}_{n}$ as

$$
M Z:=(A Z+B)(C Z+D)^{-1} \text {. }
$$

The discrete group $\Gamma$ can more generally be chosen to be a congruence subgroup $\Gamma$ of the Siegel modular group $\operatorname{Sp}(n, \mathbb{Z})$, such as the Hecke congruence $\Gamma_{0}^{(n)}(N)$ of level $N$, defined by the constraint that $C \equiv 0(\bmod N)$. The dimension of the Siegel upper halfplane $X=\mathcal{H}_{n}$ also determines the number of algebraically independent functions on $\mathcal{H}_{n}$ that are invariant under the Siegel modular group $\operatorname{Sp}(n, \mathbb{Z})$.

Generators of the space of automorphic functions with respect to the Siegel modular group of this space can be constructed in terms of the series

$$
E_{w, s}(Z)=\sum_{M \in \Gamma_{\infty}^{(n)} \backslash \operatorname{Sp}(n, \mathbb{Z})} \frac{\operatorname{det} \operatorname{Im}(M Z)^{s}}{J(M, Z)^{w}}
$$


which generalize the real-analytic Eisenstein series of genus 1 . Here $w>n+1, Z \in \mathcal{H}_{n}$, the symplectic automorphy factor $J(g, z)$ is given by

$$
J(M, Z)=\operatorname{det}(C Z+D), \quad M=\left(\begin{array}{ll}
A & B \\
C & D
\end{array}\right) \in \operatorname{Sp}(n, \mathbb{R}),
$$

and

$$
\Gamma_{\infty}^{(n)}=\left\{\left(\begin{array}{cc}
A & B \\
0 & D
\end{array}\right) \in \operatorname{Sp}(n, \mathbb{Z})\right\}
$$

For $s=0$ these functions reduce to the series considered originally by Siegel [38]. Using the notation $E_{w}=E_{w, 0}$ for these series one can consider the invariant functions

$$
F_{r s}(Z):=\frac{E_{r s}(Z)}{\left(E_{s}(Z)\right)^{r}}
$$

for $s>n+1$, which can be shown to generate Siegel modular functions on $\mathcal{H}_{n}[39,40]$.

It is also possible to consider more general Eisenstein series, following Klingen, by lifting lower rank cusp forms. These are defined with respect to certain discrete subgroups $\Gamma_{r}^{(n)} \subset \Gamma^{(n)}$ for $0 \leq r \leq n[41,42]$ and cusp forms $F$ of degree $r$, evaluated at the projection $(M Z)_{r}$ of $M Z$ via

$$
E_{w, r, F}(Z)=\sum_{M \in \Gamma_{r}^{(n)} \backslash \Gamma^{(n)}} \frac{F(M Z)_{r}}{\operatorname{det}(C Z+D)^{w}} .
$$

It is therefore possible for example to lift classical cusp forms such as the Ramanujan form $\Delta=\eta^{24}(\tau)$ (see below) to Siegel modular forms.

The Siegel Eisenstein series $E_{w}(Z), Z \in \mathcal{H}_{n}$ specialize for $n=1$ to the classical Eisenstein series of the upper halfplane. The latter were considered in the context of twofield inflation in [9]. The next case in this sequence is given by genus 2 Eisenstein series for $\operatorname{Sp}(2, \mathbb{R})$, describing models of six-field inflation. The generators of this function field were first determined by Igusa $[43,44]$ in terms of Eisenstein series of weight 4 and 6 , as well as three theta series of weight 10, 12 and 35, where the latter satisfies a polynomial equation in terms of the first 4, and the weight 10 and 12 forms can be expressed in terms of the above Eisenstein series. This led in [43] to a set of generators of the function field involving forms of quite high weight. An alternative set of generators, involving lower weight forms was given by Freitag [45] in his simpler proof of Igusa's result, leading to

$$
\frac{E_{4} E_{6}}{E_{10}}, \quad \frac{E_{6}^{2}}{E_{12}}, \quad \frac{E_{4}^{5}}{E_{10}^{2}}
$$

The same structure can be extended to other groups, for example GL $(n)$-automorphic inflation models based on $\mathrm{GL}(n, \mathbb{Z})$-invariant functions that can be obtained from Eisenstein series associated to parabolic subgroups in $\operatorname{GL}(n, \mathbb{R})$. 


\section{Inflation from modular field theory}

Over the past decade much effort has gone into the exploration of a number of different twofield inflationary models with curved target spaces, often with restrictions on the couplings of the fields, such as sum-separable or product-separable potentials. (See for example [4654] and references therein.) The specialization of automorphic field theory to two fields leads to new types of classes of highly structured potentials involving classical modular forms, leading to a stratified space of two-field inflation models with curved target spaces. As noted earlier, classical modular forms were introduced in the second half of the $19^{\text {th }}$ century as functions on the complex upper halfplane because this space is mapped to itself by the modular group $\mathrm{SL}(2, \mathbb{Z})$. Thinking about forms in this way is computationally useful, but conceptually limiting, and it is best to have both the domain function view and the group function view available.

In the context of modular field theory the group $G=\mathrm{SL}(2, \mathbb{R})$ is semisimple and the maximal compact subgroup is the rotation group $K=\mathrm{SO}(2, \mathbb{R})$, with the group action given by the Möbius transformation. Thus the $(G, K)$ structure is fixed for this class of models. The discrete groups $\Gamma$ on the other hand can be chosen from various families that are parametrized by an integer $N$, the level. The theory has been developed in most detail for the Hecke congruence group $\Gamma_{0}(N)$, defined by matrices $\gamma=\left(\begin{array}{ll}a & b \\ c & d\end{array}\right)$ in the modular group $\mathrm{SL}(2, \mathbb{Z})$ such that $c \equiv 0(\bmod N)$. Such modular forms play an important role in the context of geometric forms relevant for motivic aspects of string theory $[55,56]$. Other possibilities for $\Gamma$ include the groups $\Gamma_{1}(N)$ defined by the further constraints $a, b \equiv 1(\bmod N)$, as well as the principal congruence subgroups $\Gamma(N)$, which satisfy the constraint $\gamma \equiv \mathbf{1}(\bmod N)$, i.e. $\Gamma(N) \subset \Gamma_{1}(N) \subseteq \Gamma_{0}(N)$. More general classes of non-congruence groups can be considered as well. These will collectively be denoted as $\Gamma_{N}$ in the following. The groups $\Gamma_{N}$ thus characterize the field space of modular inflation in terms of their type $t_{\Gamma}$ as well as their level $N=N_{\Gamma}$.

\subsection{Modular potentials}

Modular forms on the group $\mathrm{SL}(2, \mathbb{R})$ are functions of the type described in section 2 . Given an arithmetic group $\Gamma_{N}$ and a character $\epsilon_{N}$ the function $\Phi$ is required to transform as $\Phi(\gamma g)=\epsilon_{N}(\gamma) \Phi(g)$ for all $\gamma \in \Gamma_{N}$ and $g \in \mathrm{SL}(2, \mathbb{R})$. The second constraint simplifies because for $k \in K$ and $g \in G$ one has

$$
\Phi(g k)=\lambda(k) \Phi(g),
$$

where $\lambda_{w}$ is the weight character $\lambda_{w}(k)=e^{i w \theta}$ for $k=\left(\begin{array}{cc}\cos \theta & \sin \theta \\ -\sin \theta & \cos \theta\end{array}\right) \in \operatorname{SO}(2, \mathbb{R})$, where $w$ is the weight of the form. In particular, the $K$-translates span a 1 -dimensional vector space. The behavior of the $G$-form with respect to the maximal compact subgroup thus introduces an additional numerical characteristic that further stratifies the scalar field theory space. This automorphic part of the data is encoded more generally by a weight representation.

The third constraint simplifies in the classical modular case because the action of the center of $\operatorname{SL}(2, \mathbb{R})$ is trivial. The final condition simplifies as well because the center of the universal enveloping algebra is one-dimensional, generated by the Casimir element 
$\mathcal{C}=h^{i j} X_{i} X_{j}$, where $h^{i j}$ is the inverse of the metric defined on the Lie algebra $\mathfrak{g}$ via the Cartan-Killing form. Thus this constraint specializes to the requirement that the function $\Phi$ is an eigenfunction of the Laplace-Beltrami operator on $\operatorname{SL}(2, \mathbb{R})$. The eigenvalue structure distinguishes between holomorphic classical modular forms and Maaß forms. These constraints can be made explicit by considering the Iwasawa decomposition $G=N A K$ of $\mathrm{SL}(2, \mathbb{R})$ in terms of the factors $N, A$ and $K$ defined above, which specialize here to

$$
\begin{aligned}
& N=\left\{\left(\begin{array}{ll}
1 & x \\
0 & 1
\end{array}\right) \mid x \in \mathbb{R}\right\} \\
& A=\left\{\left(\begin{array}{cc}
\sqrt{y} & 0 \\
0 & \sqrt{y}^{-1}
\end{array}\right) \mid y \in \mathbb{R}^{+}\right\} \\
& K=\operatorname{SO}(2, \mathbb{R}) .
\end{aligned}
$$

The motivation for the specific form of the factor $A$ comes from fact that the Möbius action of $N A$ on the $K$-isotropy point $i$ gives $N A i=x+i y=z \in \mathcal{H}$.

The Iwasawa decomposition in this case leads to $\mathrm{SL}(2, \mathbb{R}) \cong \mathbb{R} \times \mathbb{R}^{+} \times[0,2 \pi)$ and the isotropy point $x_{0}=i$ establishes the isomorphism $N A \cong \mathcal{H}$. In these coordinates the Laplace-Beltrami operator reduces on the bounded domain $X=G / K$, which is isomorphic to the upper halfplane

$$
X=\mathrm{SL}(2, \mathbb{R}) / \mathrm{SO}(2, \mathbb{R}) \cong \mathcal{H}=\{\tau \in \mathbb{C} \mid \operatorname{Im}(\tau)>0\}
$$

to

$$
\Delta=-y^{2}\left(\frac{\partial^{2}}{\partial x^{2}}+\frac{\partial^{2}}{\partial y^{2}}\right)
$$

where $\tau=x+i y$. Since the rank of $\operatorname{SL}(2, \mathbb{R})$ is one, the center of the universal enveloping algebra is spanned by the Laplacian, $Z_{\mathrm{U}}(\mathfrak{g}) \cong \mathbb{C}(\Delta)$.

Classical modular forms on $\mathcal{H}$ are induced by group functions $\Phi: \operatorname{SL}(2, \mathbb{R}) \longrightarrow \mathbb{C}$ via the 1-cocycle, given in this case by

$$
J(g, \tau)=(c \tau+d), \quad g=\left(\begin{array}{ll}
a & b \\
c & d
\end{array}\right) .
$$

This leads to the definition

$$
f(\tau)=(c \tau+d)^{w} \Phi(g),
$$

where $\tau=g i$. They are defined with respect to discrete subgroups $\Gamma_{N}$ of the modular group $G(\mathbb{Z})=\mathrm{SL}(2, \mathbb{Z})$ and are characterized by the level $N$ of these subgroups, their weight $w$, as well as a character $\epsilon_{N}$ via their transformation behavior, which for

$$
\gamma=\left(\begin{array}{ll}
a & b \\
c & d
\end{array}\right) \in \Gamma_{N}
$$

and the action of the Möbius transformation

$$
\gamma \tau=\frac{a \tau+b}{c \tau+d}
$$

leads to

$$
f(\gamma \tau)=\epsilon_{N}(d)(c \tau+d)^{w} f(\tau)
$$




\subsection{Kinetic term}

The field space metric

$$
d s^{2}=G_{I J} d \phi^{I} d \phi^{J}
$$

induced by the Cartan-Killing form $B$ on the Lie algebra $\mathfrak{s l}(2, \mathbb{R})$ is the Poincaré metric, which in terms of the dimensionless variable $\tau^{I}=\phi^{I} / \mu$ via the energy scale $\mu$ can be written with $\tau=\tau^{1}+i \tau^{2}$ as

$$
d s^{2}=\frac{d \tau d \bar{\tau}}{\operatorname{Im}(\tau)^{2}}
$$

defining a conformally flat target space geometry with

$$
G_{I J}=\left(\frac{\mu}{\phi^{2}}\right)^{2} \delta_{I J}
$$

The kinetic term then takes the form

$$
G_{I J} g^{\mu \nu} \partial_{\mu} \phi^{I} \partial_{\nu} \phi^{J}=\frac{\mu^{2}}{\operatorname{Im}(\tau)^{2}} g^{\mu \nu} \partial_{\mu} \tau \partial_{\nu} \bar{\tau}
$$

and the action of modular inflation can be written as

$$
\mathcal{A}_{\text {mod }}=\int d^{4} x \sqrt{-g}\left(\frac{M_{\mathrm{Pl}}^{2}}{2} R-\frac{\mu^{2}}{2 \operatorname{Im}(\tau)^{2}} g^{\mu \nu} \partial_{\mu} \tau \partial_{\nu} \bar{\tau}-V\left(F_{i}(\tau)\right)\right)
$$

where the functions $F_{i}(\tau)$ are modular forms of weight $w_{i}$ on $\mathcal{H}$.

\subsection{Geometry of the target space}

The Christoffel symbols of the Poincaré metric are given by $\Gamma_{11}^{1}=\Gamma_{22}^{1}=\Gamma_{12}^{2}=0$ and

$$
\Gamma_{11}^{2}=-\Gamma_{22}^{2}=-\Gamma_{12}^{1}=\frac{1}{\mu \operatorname{Im}(\tau)}
$$

The bounded domain $X=\mathcal{H}$ is two-dimensional, which implies via the Bianchi identity that the Riemann curvature tensor takes the form

$$
R_{I J K L}=K\left(G_{I K} G_{J L}-G_{I L} G_{J K}\right),
$$

where $K=R / 2$ is the Gaussian curvature expressed in terms of the Ricci scalar $R$. The Riemann tensor $R_{I J K L}$ has only one independent component

$$
R_{1212}=-\frac{\mu^{2}}{\left(\phi^{2}\right)^{4}},
$$

leading to the constant curvature scalar $R=-2 / \mu^{2}$. 


\subsection{Modular Eisenstein series}

As in the automorphic case, Eisenstein series play a key role for modular forms of arbitrary weight because for the full modular group they span the subspace complementary to the cusp forms. They also provide the building blocks for $j$-inflation [9].

Holomorphic Eisenstein series are obtained by following the general construction briefly outlined in the general case in the previous section. The ingredients of the definition are obtained as follows. The group factorization $G=P K$ into the parabolic factor and the compact factor can be obtained from

$$
P=N A=\left\{\left(\begin{array}{cc}
\sqrt{y} & \frac{x}{\sqrt{y}} \\
0 & \frac{1}{\sqrt{y}}
\end{array}\right)\right\} .
$$

The function $f_{K}: K \longrightarrow \mathbb{C}$ is determined by the automorphy factor $J(g, \tau)$ of eq. (4.5), restricted to the compact subgroup $K$ with base point $i=\sqrt{-1}$, and an integer $w$ as

$$
f_{w}(k):=\frac{1}{J(k, i)^{w}} .
$$

The lift is characterized by a single complex parameter $s \in \mathbb{C}$ as

$$
f_{s, w}(g):=\delta_{P}(p)^{s / 2} f_{w}(k),
$$

where the modulus character $\delta_{P}$ is defined for a $(2 \times 2)$ matrix with the diagonal $(a, b)$ as

$$
\delta_{P}(p)=\frac{a}{b} .
$$

Denoting the discrete subgroup by $\Gamma$ then leads to the Eisenstein series associated to $\operatorname{SL}(2, \mathbb{R})$

$$
E_{s, w}(g):=\sum_{\gamma \in(P \cap \Gamma) \backslash \Gamma} f_{s, w}(\gamma g)=\sum_{\gamma \in(P \cap \Gamma) \backslash \Gamma} \frac{\delta_{P}\left(p_{\gamma}\right)^{s / 2}}{J\left(k_{\gamma}, i\right)^{w}} .
$$

This leads to both holomorphic and real-analytic Eisenstein series, in particular the classical Eisenstein series

$$
E_{w}(\tau)=1-\frac{2 w}{B_{w}} \sum_{n \geq 1} \sigma_{w-1}(n) q^{n}
$$

where $B_{w}$ are the Bernoulli numbers, $\sigma_{w}(n)$ is the divisor function

$$
\sigma_{w}(n):=\sum_{d \mid n} d^{w}
$$

and $q=e^{2 \pi i \tau}$. These functions generate the non-cuspidal part of the space of modular forms.

\subsection{Modular functions}

Functions that are invariant with respect to subgroups $\Gamma_{N}$ of the modular group $\operatorname{SL}(2, \mathbb{Z})$ can be constructed by considering quotients of modular forms with respect to the same arithmetic group and the same weight. An important example is the quotient of two 
modular forms of weight twelve, the cube of $E_{4}$, and the Ramanujan modular form $\Delta$, which can be expressed in terms of the Eisenstein series as

$$
\Delta(\tau)=\frac{E_{4}^{3}-E_{6}^{2}}{1728}
$$

Both forms are modular with respect to the full modular group $\Gamma_{1}=\operatorname{SL}(2, \mathbb{Z})$, but contrary to the Eisenstein series, $\Delta$ is a cusp form. The latter appears in many different contexts, ranging from the bosonic partition function to the theory of motives. The quotient of these two weight 12 forms leads to the elliptic modular invariant

$$
j(\tau)=\frac{E_{4}(\tau)^{3}}{\Delta(\tau)}
$$

an example of an $\mathrm{SL}(2, \mathbb{Z})$-invariant function on $\mathcal{H}$ constructed in a simple way from Eisenstein series. This function enters a number of different applications, and an inflationary model based on $j(\tau)$ has been considered in [9].

Different types of modular forms can be constructed in a systematic way by considering modular forms for congruence subgroups $\Gamma_{N} \subset \mathrm{SL}(2, \mathbb{Z})$. One extension is obtained by defining Eisenstein series associated to such groups [57], while a second generalization involves the Dedekind eta-function

$$
\eta(q)=q^{1 / 24} \prod_{n \geq 1}\left(1-q^{n}\right),
$$

where $q=e^{2 \pi i \tau}$. The latter can be used to consider products of the form

$$
f(\tau)=\eta^{a_{1}}\left(q^{b_{1}}\right) \cdots \eta^{a_{n}}\left(q^{b_{n}}\right)
$$

for appropriate constants $a_{i}, b_{i} \in \mathbb{Z}$. This also provides an alternative construction of the Ramanujan form as $\Delta(\tau)=\eta^{24}(\tau)$. Either type of congruence modular form can be used to construct modular functions via appropriate quotients.

\section{Conclusions}

Automorphic inflation can be seen as an application of a general scalar field theory framework involving potentials constructed from highly symmetric functions that are derived from automorphic forms associated to certain types of Lie groups $G$. The defining features include the choice of a discrete subgroup $\Gamma \subset G$, examples of which are given by the class of arithmetic groups. A third ingredient is the choice of a maximal compact subgroup $K$ of $G$, determined by a Cartan involution $\theta$. With these ingredients a special class of functions is defined on $G$ whose transformation behavior induces automorphic forms on the quotient space $X=G / K A$, generalizing the usual transformation behavior via Jacobians.

The numerical characteristics of group pairs $(G, \Gamma)$ include the dimension $\operatorname{dim} G$ and the rank rk $G$ of the continuous group $G$, as well as the level $N$ of the discrete group $\Gamma_{N}$. Further data is provided by the weights $w_{i}$ of the automorphic forms $\left(\Phi_{i}, F_{i}\right)$. One can therefore think of the space of field theories as being stratified by the group 
structure $\left(G, \Gamma_{N}\right)$, in which the different strata are characterized by a lattice of integers $\left(\operatorname{dim} G, \operatorname{rk} G, N, w_{i}\right)$.

A special class of inflationary theories that has received much attention in past years is two-field inflation. Reducing automorphic field theories to this context leads to modular field theories, associated to the group of Möbius transformation $\operatorname{SL}(2, \mathbb{R})$ on the complex upper halfplane, leading to a framework for two-field inflation. Here the dimension and rank of $G$ are fixed and the field theory space is stratified by the type of discrete subgroup $\Gamma_{N}$, its level $N$, and the weights $w_{i}$ of the modular building blocks. It would be interesting to explore the effect of higher levels in either the context of modular inflation models or in the general automorphic context, for example via symplectic Eisenstein series associated to congruence subgroups of the Siegel modular group [58].

\section{Acknowledgments}

It is a pleasure to thank Monika Lynker for discussions. This work was supported in part by the NSF grant 0969875 . The author is grateful for the hospitality and support by the Simons Center for Geometry and Physics, where part of this work was done.

Open Access. This article is distributed under the terms of the Creative Commons Attribution License (CC-BY 4.0), which permits any use, distribution and reproduction in any medium, provided the original author(s) and source are credited.

\section{References}

[1] A.D. Linde, Chaotic Inflation, Phys. Lett. B 129 (1983) 177 [INSPIRE].

[2] K. Enqvist and J. Maalampi, Problems With Chaotic Inflation, Phys. Lett. B 180 (1986) 14 [INSPIRE].

[3] K. Freese, J.A. Frieman and A.V. Olinto, Natural inflation with pseudo-Nambu-Goldstone bosons, Phys. Rev. Lett. 65 (1990) 3233 [INSPIRE].

[4] F.C. Adams, J.R. Bond, K. Freese, J.A. Frieman and A.V. Olinto, Natural inflation: Particle physics models, power law spectra for large scale structure and constraints from COBE, Phys. Rev. D 47 (1993) 426 [hep-ph/9207245] [INSPIRE].

[5] E. Silverstein and A. Westphal, Monodromy in the CMB: Gravity Waves and String Inflation, Phys. Rev. D 78 (2008) 106003 [arXiv:0803.3085] [INSPIRE].

[6] L. McAllister, E. Silverstein and A. Westphal, Gravity Waves and Linear Inflation from Axion Monodromy, Phys. Rev. D 82 (2010) 046003 [arXiv:0808. 0706] [INSPIRE].

[7] E. Pajer and M. Peloso, A review of Axion Inflation in the era of Planck, Class. Quant. Grav. 30 (2013) 214002 [arXiv: 1305.3557] [inSPIRE].

[8] E. Silverstein, Les Houches lectures on inflationary observables and string theory, arXiv:1311.2312 [INSPIRE].

[9] R. Schimmrigk, Automorphic inflation, Phys. Lett. B 748 (2015) 376 [arXiv:1412.8537] [INSPIRE]. 
[10] B.A. Bassett, S. Tsujikawa and D. Wands, Inflation dynamics and reheating, Rev. Mod. Phys. 78 (2006) 537 [astro-ph/0507632] [INSPIRE].

[11] D. Wands, Multiple field inflation, Lect. Notes Phys. 738 (2008) 275 [astro-ph/0702187] [INSPIRE].

[12] C.M. Peterson and M. Tegmark, Testing multifield inflation: A geometric approach, Phys. Rev. D 87 (2013) 103507 [arXiv:1111.0927] [InSPIRE].

[13] C.P. Burgess, M.W. Horbatsch and S. Patil, Inflating in a Trough: Single-Field Effective Theory from Multiple-Field Curved Valleys, JHEP 01 (2013) 133 [arXiv:1209.5701] [INSPIRE].

[14] D.I. Kaiser, E.A. Mazenc and E.I. Sfakianakis, Primordial Bispectrum from Multifield Inflation with Nonminimal Couplings, Phys. Rev. D 87 (2013) 064004 [arXiv:1210.7487] [INSPIRE].

[15] M.A. Amin, M.P. Hertzberg, D.I. Kaiser and J. Karouby, Nonperturbative Dynamics Of Reheating After Inflation: A Review, Int. J. Mod. Phys. D 24 (2014) 1530003 [arXiv: 1410.3808] [INSPIRE].

[16] J.J. Blanco-Pillado, M. Dias, J. Frazer and K. Sousa, Large Scale Power Suppression in a Multifield Landscape, arXiv:1503.07579 [INSPIRE].

[17] Y. Watanabe and J. White, Multifield formulation of gravitational particle production after inflation, Phys. Rev. D 92 (2015) 023504 [arXiv:1503.08430] [INSPIRE].

[18] M.P. DeCross, D.I. Kaiser, A. Prabhu, C. Prescod-Weinstein and E.I. Sfakianakis, Preheating after Multifield Inflation with Nonminimal Couplings, I: Covariant Formalism and Attractor Behavior, arXiv:1510.08553 [INSPIRE].

[19] J.-O. Gong and T. Tanaka, A covariant approach to general field space metric in multi-field inflation, JCAP 03 (2011) 015 [Erratum ibid. 02 (2012) E01] [arXiv:1101.4809] [INSPIRE].

[20] F. Klein, Zur Theorie der Laméschen Funktionen, Göttinger Nachrichten 1890, vorgelegt am 11 März.

[21] S. Helgason, Differential geometry, Lie groups, and symmetric spaces, Graduate Studies in Mathematics volume 34, Am. Math. Soc. (1978).

[22] R. Godement, Generalites sur les formes modulaires II, Seminaire Henri Cartan 10 $(1957 / 58) 1$.

[23] Harish-Chandra, Automorphic forms on a semisimple Lie group, Proc. Nat. Acad. Sci. $\mathbf{4 5}$ (1959) 570.

[24] Harish-Chandra, Automorphic forms on semi-simple Lie groups, Springer (1968).

[25] A. Borel and W. Casselman, Automorphic forms, representations, and L-functions, Proc. Pure Math. volume 33, Am. Math. Soc. (1979).

[26] S. Bochner, Algebraic and linear dependence of automorphic functions in several variables, J. Indian Math. Soc. 16 (1952) 1.

[27] H. Maaß, Siegel's modular forms and Dirichlet Series, Lect. Notes Math. volume 216, Springer (1971).

[28] G. van der Geer, Siegel modular forms and their applications, in The 1-2-3 of Modular Forms, J.H. Bruinier et al. eds., Springer Universitext (2008). 
[29] L.C. Siegel, Symplectic geometry, Am. J. Math. 65 (1943) 1.

[30] H. Maaß, Über eine Metrik im Siegelschen Halbraum, Math. Ann. 118 (1942) 312.

[31] A. Sen, Microscopic and Macroscopic Entropy of Extremal Black Holes in String Theory, Gen. Rel. Grav. 46 (2014) 1711 [arXiv:1402.0109] [inSPIRE].

[32] R. Schimmrigk, Automorphic Black Hole Entropy, Mod. Phys. Lett. A 28 (2013) 1330026 [arXiv: 1312.7168] [INSPIRE].

[33] K. Cassella and R. Schimmrigk, Automorphic Black Holes as Probes of Extra Dimensions, Nucl. Phys. B 858 (2012) 317 [arXiv:1110.6077] [INSPIRE].

[34] P. Fleig, H.P.A. Gustafsson, A. Kleinschmidt and D. Persson, Eisenstein series and automorphic representations, arXiv:1511.04265 [INSPIRE].

[35] R.P. Langlands, On the functional equation satisfied by Eisenstein series, Lect. Notes. Math. volume 544, Springer (1976).

[36] R.P. Langlands, Eisenstein series, the trace formula, and the modern theory of automorphic forms, in Number Theory, Trace Formula and Discrete Groups, K.E. Aubert et al. eds., Academic Press (1989).

[37] C.L. Siegel, Über die analytische Theorie der quadratischen Formen, Ann. Math. 36 (1935) 527.

[38] C.L. Siegel, Einführung in die Theorie der Modulfunktionen n-ten Grades, Math. Ann. 116 (1939) 617.

[39] C.L. Siegel, Topics in complex function theory, volume 3, Wiley (1973).

[40] E. Freitag, Siegelsche Modulfunktionen, Grundlehren der mathematischen Wissenschaften volume 254, Springer (1983).

[41] H. Klingen, Zum Darstellungssatz für Siegelsche Modulformen, Math. Zeitschr. 102 (1967) 30.

[42] H. Klingen, Introductory lectures on Siegel modular forms, Springer (1990).

[43] J.-I. Igusa, On Siegel modular forms of genus two, Am. J. Math. 84 (1962) 175.

[44] J.-I. Igusa, On Siegel modular forms of genus two (II), Am. J. Math. 89 (1964) 392.

[45] E. Freitag, Zur Theorie der Modulformen zweiten Grades, Nachr. Akad. Wiss. Göttingen (1965) 151.

[46] C.M. Peterson and M. Tegmark, Testing Two-Field Inflation, Phys. Rev. D 83 (2011) 023522 [arXiv:1005.4056] [INSPIRE].

[47] S. Cespedes, V. Atal and G.A. Palma, On the importance of heavy fields during inflation, JCAP 05 (2012) 008 [arXiv:1201.4848] [INSPIRE].

[48] T. Higaki and F. Takahashi, Elliptic inflation: interpolating from natural inflation to $R^{2}$-inflation, JHEP 03 (2015) 129 [arXiv:1501.02354] [INSPIRE].

[49] A. Achúcarro, V. Atal and Y. Welling, On the viability of $m^{2} \phi^{2}$ and natural inflation, JCAP 07 (2015) 008 [arXiv: 1503.07486] [INSPIRE].

[50] K. Bamba, S.D. Odintsov and P.V. Tretyakov, Inflation in a conformally-invariant two-scalar-field theory with an extra $R^{2}$ term, Eur. Phys. J. C 75 (2015) 344 [arXiv: 1505.00854] [INSPIRE]. 
[51] X. Gao and J.-O. Gong, Towards general patterns of features in multi-field inflation, JHEP 08 (2015) 115 [arXiv: 1506.08894] [INSPIRE].

[52] J. Erlich, J. Olsen and Z. Wang, Multi-field inflation and the field-space metric, arXiv:1509.06781 [INSPIRE].

[53] S. Kaneda and S.V. Ketov, Starobinsky-like two-field inflation, Eur. Phys. J. C 76 (2016) 26 [arXiv: 1510.03524] [INSPIRE].

[54] A. Achúcarro, V. Atal, M. Kawasaki and F. Takahashi, The two-field regime of natural inflation, JCAP 12 (2015) 044 [arXiv:1510.08775] [INSPIRE].

[55] R. Schimmrigk, The Langlands program and string modular K3 surfaces, Nucl. Phys. B 771 (2007) 143 [hep-th/0603234] [INSPIRE].

[56] R. Schimmrigk, Emergent spacetime from modular motives, Commun. Math. Phys. 303 (2011) 1 [arXiv:0812.4450] [InSPIRE].

[57] T. Miyake, Modular forms, Springer (1989).

[58] S. Böcherer, On the space of Eisenstein series for $\Gamma_{0}(p)$ : Fourier expansions, Comment. Math. Univ. St. Paul. 63 (2014) 3. 